\title{
MENSURAÇÃO DA ESPESSURA CORNEANA EM OVINOS COM PAQUIMETRIA ULTRA-SÔNICA E MICROSCOPIA ESPECULAR DE NÃO CONTATO
}

\author{
(Corneal thickness measurements in ovines with ultrasonic pachymetry \\ and noncontact specular microscopy)
}

\author{
BRANDÃO, C.V.S.. ${ }^{1}$ RANZANI, J.J.T. ${ }^{1}$; RODRIGUES, G.N. ${ }^{2}$; MARINHO, L.F.L.P. ${ }^{2}$; PEIXOTO, T.P. ${ }^{2}$; \\ TEIXEIRA, C.R. ${ }^{3}$; CREMONINI, D.N. ${ }^{2}$; LIMA, L.S.A. ${ }^{2}$; CHIURCIU, J.L.V. ${ }^{2}$
}

\begin{abstract}
'Departamento de Cirurgia e Anestesiologia Veterinárias - FMVZ - Unesp - Botucatu; ${ }^{2}$ Pós-graduandos em Medicina Veterinária - área de Cirurgia - FMVZ - Unesp - Botucatu;

${ }^{3}$ Departamento de Cirurgia e Anestesiologia Veterinárias - FMVZ - Unesp - Botucatu.
\end{abstract}

\begin{abstract}
RESUMO - O propósito do presente trabalho foi avaliar os valores da espessura corneana central em córneas de ovinos sadios com o microscópio especular de nãocontato e paquímetro ultra-sônico. A espessura corneana central foi determinada em 22 olhos de 11 ovinos, inicialmente com microscópio especular de nãocontato (Topcon SP-2000P), seguido pelo paquímetro ultra-sônico (Nidek UP-1000). Os valores médios das medidas corneanas centrais foram $654 \mathrm{~mm}$ e $665 \mathrm{~mm}$ com o microscópio especular de não contato e o paquímetro ultra-sônico, respectivamente. As medidas da espessura corneana demonstraram que ambos os instrumentos são confiáveis.
\end{abstract}

Palavras-chave: Ovino. Paquimetria. Especular. Ultrasônica. Espessura corneana.

ABSTRACT - The purpose of this study was to evaluate the central corneal thickness values in normal ovine with Topcon SP-2000P noncontact specular microscope and ultrasonic pachymeter. Central corneal thickness was detemined in 22 eyes of 11 ovines, first with a noncontact specular microscopy (Topcon SP-2000P), then using an ultrasonic pachgmeter (Nidek UP-1000). The central corneal measurements were $654 \mathrm{~mm}$ e $665 \mathrm{~mm}$ with Topcon and ultrasonic pachymeter, respectively. The instruments were reliable in their measurements.

Key-words: Ovine. Pachymetry. Specular. Ultrasonic. Corneal thickness.

\section{Introdução}

A avaliação da espessura corneana central é importante em uma grande variedade de afecções oculares (POLE e BATZER, 1985; LIU e FLUGFELDER, 1999; LANGSTON, 2001) tais como glaucoma (COPT et al., 1999; BRANDT et al., 2001; HERMAN et al., 2001), olho seco e diabetes mellitus (SAINI e MITTAL, 1996).
A microscopia especular e ultra-sônica são métodos aceitos de mensuração da espessura corneana (WHEELER et al., 1992; BOVELLE et al., 1999; YAMANE, 2003; OGBUEHI e ALMUBRAD, 2005a; OGBUEHI e ALMUBRAD, 2005b), podendo ser obtidas após a reflexão da luz ou ondas ultra-sônicas da superfície anterior e posterior da córnea. O microscópio especular de não contato é capaz de avaliar o padrão endotelial e realizar a paquimetria corneana ao mesmo tempo. Fotografando o endotélio, este equipamento gera imagem especular e medidas de distância focal, possibilitando o cálculo da espessura corneana (SANCHIS-GIMENO et al., 2006).

$\mathrm{Na}$ literatura veterinária consultada, não foram observados estudos de paquimetria por microscopia especular em ovinos, ou a comparação desta com a ultra-sônica. O objetivo deste estudo foi determinar e comparar a espessura corneana central de ovinos sadios, obtida com paquímetro ultra-sônico e microscópio especular de não contato.

\section{Material e Método}

Foram utilizados 22 olhos normais, provenientes de 11 ovinos mestiços da raça lle de France com um ano de idade, sendo seis fêmeas e cinco machos. Todos foram submetidos a exame oftalmológico para a exclusão de animais com alterações oculares. As medidas da paquimetria foram realizadas imediatamente após o abate dos animais, os quais foram destinados ao consumo humano.

A espessura central corneana foi mensurada por meio de microscopia especular de não-contato no modo automático. Para a paquimetria ultra-sônica, um probe de $20 \mathrm{MHz}$ foi posicionado perpendicularmente à superfície corneana central; foram realizados um total de cinco medidas, sendo a média das mesmas consideradas o valor de paquimetria central de cada animal. A comparação entre grupos foi realizada pelo teste de Wilcoxon pareado.

Autor para correspondência: Claudia Valéria Seullner Brandão - Departamento de Cirurgia e Anestesiologia Veterinárias - Faculdade de Medicina Veterinária e Zootecnia da UNESP - Distrito de Rubião Júnior s/n - 18618-000 - Botucatu-SP - E-mail:valeriasb@fmvz.unesp.br. 
Mensuração da espessura corneana em ovinos com paquimetria ultra-sônica e microscopia...

\section{Resultados e Discusão}

Os dados médios de espessura corneana central foram de $654.09 \pm 8.3 \mathrm{~mm}$ com o microscópio especular e de $665.82 \pm 82 \mathrm{~mm}$, com o paquímetro ultra-sônico. Em humanos, espessuras de $542 \pm 46$ e $570 \pm 42 \mathrm{~mm}$ são descritas (DOUGHTY e ZAMAN, 2000; MODIS et al., 2001) enquanto que em cães, os valores descritos são ao redor de 600mm (GILGER et al., 1991; KUDO et al., 1996; AZEVEDO, 2001).

Estatisticamente, não foi verificada diferença significativa entre os dois métodos, diferentemente do descrito por MODIS et al. (2001) e WHEELER et al. (1992), os quais relataram medidas de paquimetria por microscopia especular de não-contato, significantemente inferiores aos resultados ultra-sônicos. Apesar da ausência da diferença estatística, os valores de paquimetria ultra-sônica obtidos foram cerca de 10 $\mathrm{mm}$ superiores em relação à microscopia especular.

No presente estudo, verificou-se ainda ausência de correlação estatística significativa entre os sexos, representados por $639 \mathrm{~mm}$ nas fêmeas e $671 \mathrm{~mm}$ nos machos.

Segundo alguns autores, os aparelhos são confiáveis em suas medidas, mas seus dados não podem ser intercambiáveis (MODIS et al., 2001; WHEELER et al., 1992). O microscópio especular também promove informações a respeito do status da córnea, tais como densidade e morfologia celular. Em casos de córneas opacas ou semitransparentes, a captura da imagem é difícil ou impossível, representando a limitação de microscópios especulares (MODIS et al., 2001).

Aparelhos de paquimetria de contato requerem anestesia tópica e contato corneano com possível risco de defeito epitelial e infecção iatrogênica; nestes, a espessura corneana central pode ser avaliada rapidamente na maioria dos casos.

\section{Conclusões}

Conclui-se que os valores médios da espessura corneana central de ovinos foram de $654 \mathrm{~mm}$ utilizando microscópio especular de não contato e $665 \mathrm{~mm}$ com o paquímetro ultra-sônico, sendo os mesmos considerados fidedignos na mensuração da espessura corneana, não demonstrando diferença estatística entre eles.

\section{Referências}

AZEVEDO, A.B. Mensurações do segmento anterior do bulbo ocular do cão. Botucatu, 2001.92p. Dissertação (Mestrado em Cirurgia Veterinária) - Faculdade de Medicina Veterinária e Zootecnia, Universidade Estadual Paulista.

BOVELLE, R.; KAUFMAN, S.C.; THOMPSON, H.W.; HIRAKU, H. Corneal thickness measurements with the Topcon SP-200P Specular Microscope and an ultrasound pachymeter. Archives of Ophthalmology, v. 117, n. 7, p. 868-870, 1999.
BRANDT, J.D.; BAISER, J.A.; KASS, M.A.; GORDON, M.O. Central corneal thickness in the Ocular Hypertension Treatment Study (OHTS). Ophthalmology, v.108, p. 1779-1788, 2001.

COPT, R.; THOMAS, R.; MERMOUD, A. Corneal thickness in ocular hypertension, primary open-angle glaucoma, and normal tension glaucoma. Archives of Ophthalmology, v.117, p.14-17, 1999.

DOUGHTY, M.J.; ZAMAN, M.L. Human corneal thickness and its impact on intraocular pressure measures: a review and meta-analysis approach. Survey of Ophthalmology, v.44, p.367-408, 2000.

GILGER, B.C.; WHITLEY, R.D.; MCLAGHLIN, S.A.; WRIEHT, J.C.; DRANE, J.W. Canine corneal thickness measured by ultrasonic pachymetry. American Journal of Veterinary Research, v.52, n.10, p.1570-1572, 1991.

HERMAN, D.C.; HODGE, D.O.; BOURNE, W.M. Increased corneal thickness in patients with ocular hypertension. Archives of Ophthalmology, v.119, p.334-336, 2001.

KUDO, S.; KANEMAKI, N.; VCHIUMI, M.; MATSUVRA, K. The thickness of the cornea using an ultrasonic pachymeter in adult dogs. Journal of the Japan Veterinary Medical Association, v.49, n.1, p.32-34, 1996.

LANGSTON, D.P. Técnicas de Exame Oftalmológico e Testes Diagnósticos. In: LANGSTON, D.P. Manual de Oftalmologia: Diagnóstico e Tratamento. 4 ed. Rio de Janeiro: Médica e Cientifica, 2001, p.1-42.

LIU, Z.; FLUGFELDER, S.C. Corneal thickness is reduced in dry eye. Cornea, v.18, p.403-407, 1999.

MODIS, L.; LANGENBUCHER, A.; SEITZ, B. Corneal thickness measurements with contact and noncontact specular microscopic and ultrasonic pachymetry. American Journal Ophthalmology, v.132, p.517-521, 2001.

OGBUEHI, K.C.; ALMUBRAD, T.M. Repeatability of central corneal thickness measurements measured with the Topcon SP2000P specular microscope. Graefes Archive for Clinical and Experimental Ophthalmology, v.243, n.8, p.798-802, 2005a.

OGBUEHI, K.C.; ALMUBRAD, T.M. Limits of agreement between the optical pachymeter and a noncontact specular microscope. Cornea. v.24, n.5, p.245-249, 2005b.

POLE, J.J.; BATZER, J.K. Corneal thickness of patients with dry eyes. Journal of the American Otometric Association, v.56, p.220-221, 1985.

SAINI, J.S.; MITTAL, S. In vivo assessment of corneal endothelial function in diabetes mellitus. Archives of Ophthalmology, v.114, p.649-653, 1996.

SANCHIS-GIMENO, J.A.; HERRERA, M.; LLEO-PEREZ, A.; ALONSO, L.; RAHHAL, M.S.; MARTINEZ-SORIANO, $F$. Quantitative anatomical differences in central corneal thickness values determined with scanning-slit corneal topography and noncontact specular microscopy. Cornea. v.25, n.2, p.203-205, 2006. 
WHEELER, N.C. et al. Reability coefficients of three corneal pachymeters. American Journal Ophthalmology, v.113, p.645-651, 1992.
YAMANE, R. Curvatura, paquimetria e microscopia especular da córnea. In: YAMANE, R. Semiologia Ocular. 1 ed. Rio de Janeiro: Cultura Médica, 2003, p.89-102.

Recebido para publicação: Aprovado:

$30 / 11 / 2005$

01/06/2006 